\title{
Blow-Up of Solutions to a Novel Two-Component Rod System
}

\author{
Shengrong Liu, Zhengguang Guo, and Weiming Wang \\ College of Mathematics and Information Science, Wenzhou University, Wenzhou 325035, China \\ Correspondence should be addressed to Weiming Wang; weimingwang2003@163.com
}

Received 4 February 2013; Accepted 23 May 2013

Academic Editor: Ziemowit Popowicz

Copyright (C) 2013 Shengrong Liu et al. This is an open access article distributed under the Creative Commons Attribution License, which permits unrestricted use, distribution, and reproduction in any medium, provided the original work is properly cited.

We consider a novel two-component rod system which is closely connected to the shallow water theory. The present work is mainly concerned with the blow-up mechanism of strong solutions; we establish new conditions in view of some special classes of initial value to guarantee finite time blow-up of solutions.

\section{Introduction}

We consider the following variation of the two-component rod system:

$$
\begin{array}{r}
u_{t}-u_{x x t}+3 u u_{x}-\sigma\left(2 u_{x} u_{x x}+u u_{x x x}\right)+\pi(\rho) \rho_{x}=0, \\
t>0, \quad x \in \mathbb{S}, \\
\pi(\rho)_{t}+(\pi(\rho) u)_{x}=0, \quad t>0, \quad x \in \mathbb{S} .
\end{array}
$$

If we introduce a momentum $y=u-u_{x x}$, the previous system possesses the following form:

$$
\begin{array}{r}
y_{t}+\sigma y_{x} u+2 \sigma y u_{x}-3(\sigma-1) u u_{x}+\pi(\rho) \rho_{x}=0, \\
t>0, x \in \mathbb{S}, \\
\pi(\rho)_{t}+(\pi(\rho) u)_{x}=0, \quad t>0, x \in \mathbb{S},
\end{array}
$$

where

$$
\pi(\rho)=\rho-\mu(\rho)=\rho-\int_{0}^{1} \rho d x,
$$

with $u(x, t)$ and $\rho(x, t)$ depending on a space variable $x \in$ $\mathbb{S}=\mathbb{R} / \mathbb{Z}$ and a time variable $t$. It is obvious that system (1) for $\pi(\rho)=0$ reduces to the rod equation studied in [112]. Particularly, for $\pi(\rho)=0$ and $\sigma=1$, (1) becomes the celebrated Camassa-Holm equation which was investigated by many authors [13-21]. It reduces to the two-component rod system for $\mu(\rho)=0$ studied in [22]. Moreover, system (1) reduces to the two-component Camassa-Holm $(\mathrm{CH} 2)$ equation for $\mu(\rho)=0$ and $\sigma=1$ which was investigated in [23-30]. The two-component rod system which includes both velocity and density variables in the dynamics possesses the following form:

$$
\begin{gathered}
u_{t}-u_{x x t}-A u_{x}+3 u u_{x}-\sigma\left(2 u_{x} u_{x x}+u u_{x x x}\right)+\rho \rho_{x}=0, \\
t>0, \quad x \in \mathbb{R} \\
\rho_{t}+(\rho u)_{x}=0, \quad t>0, x \in \mathbb{R} .
\end{gathered}
$$

We note that the geometric structure of (1) is different from (4) with the incorporation of the term $\mu(\rho)$; this restricts our discussion only on the unit circle. Furthermore, the discussion of this work shows that system (1) possesses wave breaking phenomenon which is described with different blow-up criteria, while system (4) admits not only breaking wave solutions but also global in time solutions. However, we do not know whether the global solutions of (1) exist or not for the time being. It is the structure of (1) that breaks some properties which previously holds for (4). It is also known that for (4)

$$
\frac{d}{d t} \int_{\mathbb{S}} \rho d x=-\int_{\mathbb{S}}(\rho u)_{x} d x=0
$$

which follows that $\int_{\mathbb{S}} \rho d x$ is an invariant with respect to time. Particularly, if $\rho$ has zero mean initially, then the solution $\rho$ to (4) will preserve zero mean for all time. This motivates us to introduce the term $\pi(\rho)$ since it always has zero mean. 
On the other hand, we consider the system (1) in the spaces $H^{s} \times H^{s-1} / \mathbb{R}$ for $s>5 / 2$ on the circle, where $H^{s}=H^{s}(\mathbb{S})$ denotes the $L^{2}$-Sobolev space of regularity, and denote by $H^{s-1} / \mathbb{R}$ the space $H^{s-1}$ with two functions being identified if they differ by a constant. The basic idea of this variation is the decomposition of $\rho \in H^{s-1}$ into $\pi(\rho)$ and $\mu(\rho)$, where $\mu(\rho) \in \mathbb{R}$ is independent on variable $x, \pi(\rho)$ belongs to $\widehat{H}^{s-1}$, a subspace of $H^{s-1}$ containing all zero mean functions. The main purpose of our work is to investigate formation of singularities of solutions to (1) where the conservation laws play crucial roles. The idea is motivated by Guo's recent works $[28,31]$.

The rest of this paper is organized as follows. In Section 2, we recall the local well-posedness theorem and show some auxiliary results which will be used in the sequel. In Section 3, the detailed blow-up criteria are established via various initial conditions and as a byproduct, the blow-up rate is presented.

\section{Preliminaries}

In this section, we would like to list some useful results for later use. We now provide the framework in which we shall reformulate (1). Let

$$
G(x)=\frac{\cosh (x-[x]-(1 / 2))}{2 \sinh (1 / 2)}, \quad x \in \mathbb{R} .
$$

Then $\left(1-\partial_{x}^{2}\right)^{-1} f=G * f$ for all $f \in L^{2}(\mathbb{S})$ and $G * y=u$, where $*$ is the spatial convolution. With this in hand, we rewrite (1) as follows:

$$
\begin{gathered}
u_{t}+\sigma u u_{x}+\partial_{x}\left(G *\left(\frac{3-\sigma}{2} u^{2}+\frac{\sigma}{2} u_{x}^{2}+\frac{1}{2} \pi(\rho)^{2}\right)\right)=0 \\
t>0, \quad x \in \mathbb{S}, \\
\pi(\rho)_{t}+(\pi(\rho) u)_{x}=0, \quad t>0, x \in \mathbb{S} .
\end{gathered}
$$

Firstly, we recall the elementary result on the local wellposedness theorem for system (7) which was shown in [32] from the geometric formulation.

Theorem 1. Let $s>5 / 2$. There is an open neighborhood $U$ containing $(0, \pi(0)) \in H^{s} \times H^{s-1} / \mathbb{R}$ such that for any $\left(u_{0}, \pi\left(\rho_{0}\right)\right) \in U$ there exists a maximal $T>0$ and a unique solution $(u, \pi(\rho))$ to the initial value problem for system (7) with

$$
\begin{aligned}
(u, \pi(\rho)) \in & C\left([0, T) ; H^{s} \times \frac{H^{s-1}}{\mathbb{R}}\right) \\
& \cap C^{1}\left([0, T) ; H^{s-1} \times \frac{H^{s-2}}{\mathbb{R}}\right),
\end{aligned}
$$

$(u, \pi(\rho))(0)=\left(u_{0}, \pi\left(\rho_{0}\right)\right)$. Moreover, the solution depends continuously on the initial data, that is, the mapping

$$
\begin{aligned}
\left(u_{0}, \pi\left(\rho_{0}\right)\right) \longmapsto(u, \pi(\rho)): U & \longrightarrow C\left([0, T) ; H^{s} \times \frac{H^{s-1}}{\mathbb{R}}\right) \\
& \cap C^{1}\left([0, T) ; H^{s-1} \times \frac{H^{s-2}}{\mathbb{R}}\right)
\end{aligned}
$$

is continuous.

We now introduce two characteristics for (7)

$$
\begin{aligned}
& \frac{\partial q_{1}}{\partial t}=u\left(q_{1}, t\right), \quad 0<t<T, x \in \mathbb{R}, \\
& q_{1}(x, 0)=x, \quad x \in \mathbb{R}, \\
& \frac{\partial q_{2}}{\partial t}=\sigma u\left(q_{2}, t\right), \quad 0<t<T, \quad x \in \mathbb{R}, \\
& q_{2}(x, 0)=x, \quad x \in \mathbb{R},
\end{aligned}
$$

where $u$ denotes the first component of the solution to (7) with certain initial data and $T$ is the lifespan of the solution, then $q_{i}(i=1,2)$ is a diffeomorphism of the line. These two characteristics are both increasing diffeomorphisms of $\mathbb{R}$. A direct calculation shows

$$
\begin{gathered}
q_{1, t x}(x, t)=u_{x}\left(q_{1}(x, t), t\right) q_{1, x}(x, t), \\
q_{2, t x}(x, t)=\sigma u_{x}\left(q_{2}(x, t), t\right) q_{2, x}(x, t) .
\end{gathered}
$$

Thus, we have

$$
q_{1, x}(x, t)=\exp \left(\int_{0}^{t} u_{x}\left(q_{1}, s\right) d s\right)>0, \quad(x, t) \in \mathbb{R} \times[0, T),
$$

$$
\begin{array}{r}
q_{2, x}(x, t)=\exp \left(\int_{0}^{t} \sigma u_{x}\left(q_{2}, s\right) d s\right)>0, \\
(x, t) \in \mathbb{R} \times[0, T) .
\end{array}
$$

This is usually called the particle trajectory method, and it is important in the discussion of blow-up phenomena. We are now in a position to state the following.

Lemma 2. Let $X_{0}=\left(u_{0}, \pi\left(\rho_{0}\right)\right) \in H^{s} \times H^{s-1} / \mathbb{R}, s>5 / 2$, and let $T$ be the maximal existence time of the solution $X=$ $(u, \pi(\rho))$ to $(7)$ with the initial data $X_{0}$. Then for all $(x, t) \in$ $\mathbb{S} \times[0, T)$, we have

$$
\pi\left(\rho\left(q_{1}, t\right)\right) q_{1, x}(x, t)=\pi\left(\rho_{0}(x)\right) .
$$


Proof. Differentiating the left-hand side of (15) with respect to $t$, we obtain

$$
\begin{aligned}
\frac{d}{d t}[\pi & \left.\left(\rho\left(q_{1}, t\right)\right) q_{1, x}(x, t)\right] \\
= & {\left[\pi_{t}\left(\rho\left(q_{1}, t\right)\right)+\pi(\rho)_{x} q_{1, t}\right] q_{1, x}(x, t) } \\
& +\pi\left(\rho\left(q_{1}, t\right)\right) q_{1, x t}(x, t) \\
= & {\left[\pi_{t}\left(\rho\left(q_{1}, t\right)\right)+\pi(\rho)_{x} u\left(q_{1}, t\right)\right] q_{1, x}(x, t) } \\
& +\pi\left(\rho\left(q_{1}, t\right)\right) u_{x}\left(q_{1}, t\right) q_{1, x}(x, t) \\
= & -(\pi(\rho) u)_{x} q_{1, x}(x, t)+\pi(\rho)_{x} u\left(q_{1}, t\right) q_{1, x}(x, t) \\
& +\pi\left(\rho\left(q_{1}, t\right)\right) u_{x}\left(q_{1}, t\right) q_{1, x}(x, t) \\
= & 0,
\end{aligned}
$$

where we have used $q_{1}$ defined in (10) and the second equation of system $(7)$. Then $\pi\left(\rho\left(q_{1}, t\right)\right) q_{1, x}(x, t)$ is independent on time $t$. Now we choose $t=0$, due to $(13)$ we know $q_{1, x}(x, 0)=$ 1. Therefore, this lemma is easily proved.

The application of (15) leads to the following result.

Theorem 3. Let $X_{0}=\left(u_{0}, \pi\left(\rho_{0}\right)\right) \in H^{s} \times H^{s-1} / \mathbb{R}, s>5 / 2$, and let $T$ be the maximal existence time of the solution $X=$ $(u, \pi(\rho))$ to $(7)$ with the initial data $X_{0}$. If there exists $M>0$ such that

$$
\left\|u_{x}(\cdot, t)\right\|_{L^{\infty}}+\left\|\pi(\rho)_{x}(\cdot, t)\right\|_{L^{\infty}} \leq M
$$

then $H^{s} \times H^{s-1}$-norm of $X$ does not blow up on $[0, T)$.

Proof. Applying the operator $\Lambda^{s}$ where $\Lambda:=\left(1-\partial_{x}^{2}\right)^{1 / 2}$ to the first equation in (7), and multiplying by $\Lambda^{s} u$, then integrating over $\mathbb{S}$, we obtain

$$
\frac{d}{d t}\|u\|_{H^{s}}^{2}=-2\left(\sigma u u_{x}, u\right)_{s}-2\left(u, f_{1}(u)\right)_{s}-2\left(u, f_{2}(u)\right)_{s}
$$

where

$$
\begin{aligned}
& f_{1}(u)=\partial_{x}\left(G * \frac{3-\sigma}{2} u^{2}\right) \\
& f_{2}(u)=\frac{1}{2} \partial_{x}\left(G *\left(\sigma u_{x}^{2}+\pi(\rho)^{2}\right)\right) .
\end{aligned}
$$

From the proof in [25], we get

$$
\begin{aligned}
& \left|\left(\sigma u u_{x}, u\right)_{s}\right| \leq c\left\|u_{x}\right\|_{L^{\infty}}\|u\|_{H^{s}}^{2}, \\
& \left|\left(u, f_{1}(u)\right)_{s}\right| \leq c\left\|u_{x}\right\|_{L^{\infty}}\|u\|_{H^{s}}^{2} .
\end{aligned}
$$

For the third term on the right-hand side of (13), we estimate it as follows:

$$
\begin{aligned}
\left|\left(u, f_{2}(u)\right)_{s}\right| \leq & \left\|f_{2}(u)\right\|_{H^{s}}\|u\|_{H^{s}} \\
\leq & c\left(\left\|u_{x}^{2}\right\|_{H^{s-1}}+\left\|\pi(\rho)^{2}\right\|_{H^{s-1}}\right)\|u\|_{H^{s}} \\
\leq & c\left(\left\|u_{x}\right\|_{L^{\infty}}\left\|u_{x}\right\|_{H^{s-1}}\right. \\
& \left.+\|\pi(\rho)\|_{L^{\infty}}\|\pi(\rho)\|_{H^{s-1}}\right)\|u\|_{H^{s}} \\
\leq & c\left(\left\|u_{x}\right\|_{L^{\infty}}+\|\pi(\rho)\|_{L^{\infty}}\right) \\
& \times\left(\|u\|_{H^{s}}^{2}+\|\pi(\rho)\|_{H^{s-1}}^{2}\right) .
\end{aligned}
$$

Combining the previous inequalities, we can get

$$
\begin{aligned}
\frac{d}{d t}\|u\|_{H^{s}}^{2} \leq & c\left(\left\|u_{x}\right\|_{L^{\infty}}+\|\pi(\rho)\|_{L^{\infty}}\right) \\
& \times\left(\|u\|_{H^{s}}^{2}+\|\pi(\rho)\|_{H^{s-1}}^{2}\right),
\end{aligned}
$$

where the constant $c$ may be different from instance to instance. We do similar estimates for the second component by applying the operator $\Lambda^{s-1}$ to the second equation in (7), and multiplying by $\Lambda^{s-1} \pi(\rho)$, then integrating over $\mathbb{S}$ to obtain

$$
\begin{aligned}
\frac{d}{d t}\|u\|_{H^{s}}^{2}= & -2\left(\pi(\rho)_{x} u, \pi(\rho)\right)_{s-1} \\
& -2\left(\pi(\rho) u_{x}, \pi(\rho)\right)_{s-1} .
\end{aligned}
$$

Similarly, we have

$$
\begin{aligned}
& \left|\left(\pi(\rho)_{x} u, \pi(\rho)\right)_{s-1}\right| \\
& \quad \leq c\left(\left\|u_{x}\right\|_{L^{\infty}}+\left\|\pi(\rho)_{x}\right\|_{L^{\infty}}\right)\left(\|u\|_{H^{s}}^{2}+\|\pi(\rho)\|_{H^{s-1}}^{2}\right), \\
& \left|\left(\pi(\rho) u_{x}, \pi(\rho)\right)_{s-1}\right| \\
& \quad \leq c\left(\left\|u_{x}\right\|_{L^{\infty}}+\|\pi(\rho)\|_{L^{\infty}}\right)\left(\|u\|_{H^{s}}^{2}+\|\pi(\rho)\|_{H^{s-1}}^{2}\right) .
\end{aligned}
$$

Therefore, it follows that

$$
\begin{aligned}
\frac{d}{d t}\|\pi(\rho)\|_{H^{s-1}}^{2} \leq & c\left(\left\|u_{x}\right\|_{L^{\infty}}+\left\|\pi(\rho)_{x}\right\|_{L^{\infty}}+\|\pi(\rho)\|_{L^{\infty}}\right) \\
& \times\left(\|u\|_{H^{s}}^{2}+\|\pi(\rho)\|_{H^{s-1}}^{2}\right) .
\end{aligned}
$$

By (22) and (25) we have

$$
\begin{aligned}
\frac{d}{d t}\left(\|u\|_{H^{s}}^{2}+\|\pi(\rho)\|_{H^{s-1}}^{2}\right) \leq & c\left(\left\|u_{x}\right\|_{L^{\infty}}+\left\|\pi(\rho)_{x}\right\|_{L^{\infty}}\right) \\
& \times\left(\|u\|_{H^{s}}^{2}+\|\pi(\rho)\|_{H^{s-1}}^{2}\right) .
\end{aligned}
$$

It is easy to obtain by Gronwall's inequality, Lemma 2, and (13) that

$$
\begin{aligned}
\|u\|_{H^{s}}^{2} & +\|\pi(\rho)\|_{H^{s-1}}^{2} \\
& \leq \exp (c M t)\left(\left\|u_{0}\right\|_{H^{s}}^{2}+\left\|\pi\left(\rho_{0}\right)\right\|_{H^{s-1}}^{2}\right) .
\end{aligned}
$$

Then the $H^{s} \times H^{s-1}$-norm of $X$ does not blow up on $[0, T)$. 
Next we present the precise blow-up scenario for sufficiently regular solutions to (7).

Theorem 4. Let $X_{0}=\left(u_{0}, \pi\left(\rho_{0}\right)\right) \in H^{s} \times H^{s-1} / \mathbb{R}, s>5 / 2$, and let $T$ be the maximal existence time of the solution $X=$ $(u, \pi(\rho))$ to $(7)$ with the initial data $X_{0}$. Then the solution $X$ blows up in finite time if and only if

$$
\begin{aligned}
& \liminf _{t \rightarrow T}\left\{u_{x}(x, t)\right\}=-\infty \text { or } \\
& \lim _{t \rightarrow T} \sup \left\{\left\|\rho_{x}(\cdot, t)\right\|_{L^{\infty}}\right\}=+\infty .
\end{aligned}
$$

Proof. Multiplying the first equation in (2) by $y$ and integrating by parts, we get

$$
\begin{aligned}
\int_{\mathbb{S}} y y_{t} d x+\sigma \int_{\mathbb{S}} y y_{x} u d x+2 \sigma \int_{\mathbb{S}} y^{2} u_{x} d x \\
-\frac{3(\sigma-1)}{2} \int_{\mathbb{S}} y\left(u^{2}\right)_{x} d x+\int_{\mathbb{S}} y \pi(\rho) \pi(\rho)_{x} d x=0 .
\end{aligned}
$$

It then follows that

$$
\begin{aligned}
\frac{1}{2} \frac{d}{d t} \int_{\mathbb{S}} y^{2} d x= & -\frac{3 \sigma}{2} \int_{\mathbb{S}} y^{2} u_{x} d x-3(\sigma-1) \int_{\mathbb{S}} u u_{x} u_{x x} d x \\
& +\frac{1}{2} \int_{\mathbb{S}} \pi(\rho)^{2} y_{x} d x \\
= & -\frac{3 \sigma}{2} \int_{\mathbb{S}} y^{2} u_{x} d x-3(\sigma-1) \int_{\mathbb{S}} u u_{x} u_{x x} d x \\
& +\frac{1}{2} \int_{\mathbb{S}} \pi(\rho)^{2} u_{x} d x-\frac{1}{2} \int_{\mathbb{S}} \pi(\rho)^{2} u_{x x x} d x
\end{aligned}
$$

Differentiating the first equation in (2) with respect to $x$, multiplying by $y_{x}$, and integrating by parts yield

$$
\begin{aligned}
\frac{1}{2} \frac{d}{d t} \int_{\mathbb{S}} y_{x}^{2} d x= & -\frac{5 \sigma}{2} \int_{\mathbb{S}} u_{x} y_{x}^{2} d x+\sigma \int_{\mathbb{S}} y^{2} u_{x} d x \\
& +\frac{3(\sigma-1)}{2} \int_{\mathbb{S}}\left(u^{2}\right)_{x x} y_{x} d x \\
& +\int_{\mathbb{S}} u_{x x x}\left(\pi(\rho)_{x}^{2}\right. \\
& \left.+\pi(\rho) \pi(\rho)_{x x}-\frac{1}{2} \pi(\rho)^{2}\right) d x .
\end{aligned}
$$

Combining (30) and (31) together, we obtain

$$
\begin{aligned}
& \frac{d}{d t} \int_{\mathbb{S}}\left(y^{2}+y_{x}^{2}\right) d x \\
& =\int_{\mathbb{S}}\left(\pi(\rho)^{2}-5 \sigma y_{x}^{2}-\sigma y^{2}\right) u_{x} d x \\
& \quad+3(\sigma-1) \int_{\mathbb{S}} u_{x}\left(2 u_{x}^{2}+5 u_{x x}^{2}\right) d x \\
& \quad+2 \int_{\mathbb{S}} u_{x x x}\left(\pi(\rho)_{x}^{2}+\pi(\rho) \pi(\rho)_{x x}-\pi(\rho)^{2}\right) d x
\end{aligned}
$$

Similar arguments made on the second equation in (2) yield

$$
\begin{gathered}
\frac{d}{d t} \int_{\mathbb{S}} \pi(\rho)^{2} d x=-\int_{\mathbb{S}} \pi(\rho)^{2} u_{x} d x \\
\frac{d}{d t} \int_{\mathbb{S}} \pi(\rho)_{x}^{2} d x=-3 \int_{\mathbb{S}} \pi(\rho)_{x}^{2} u_{x} d x \\
+\int_{\mathbb{S}} \pi(\rho)^{2} u_{x x x} d x \\
\frac{d}{d t} \int_{\mathbb{S}} \pi(\rho)_{x x}^{2} d x=-5 \int_{\mathbb{S}} \pi(\rho)_{x x}^{2} u_{x} d x \\
-\int_{\mathbb{S}}\left(2 \pi(\rho) \pi(\rho)_{x x}-3 \pi(\rho)_{x}^{2}\right) u_{x x x} d x
\end{gathered}
$$

It follows by combining (32) and (33) that

$$
\begin{gathered}
\frac{d}{d t} \int_{\mathbb{S}}\left(y^{2}+y_{x}^{2}+\pi(\rho)^{2}+\pi(\rho)_{x}^{2}+\pi(\rho)_{x x}^{2}\right) d x \\
=-\int_{\mathbb{S}} u_{x}\left[\sigma y^{2}+5 \sigma y_{x}^{2}+3 \pi(\rho)_{x}^{2}+5 \pi(\rho)_{x x}^{2}\right. \\
\left.+3(1-\sigma)\left(2 u_{x}^{2}+5 u_{x x}^{2}\right)\right] d x \\
+\int_{\mathbb{S}} u_{x x x}\left(5 \pi(\rho)_{x}^{2}-\pi(\rho)^{2}\right) d x
\end{gathered}
$$

Assume that there exist $M_{1}>0$ and $M_{2}>0$, such that

$$
\begin{gathered}
u_{x}(x, t) \geq-M_{1}, \quad(x, t) \in \mathbb{S} \times[0, T), \\
\left\|\pi(\rho)_{x}\right\|_{L^{\infty}}=\left\|\rho_{x}\right\|_{L^{\infty}} \leq M_{2}, \quad \forall t \in[0, T) .
\end{gathered}
$$

By Lemma 2, we have

$$
\|\pi(\rho)\|_{L^{\infty}} \leq e^{M_{1} T}\left\|\pi\left(\rho_{0}\right)\right\|_{L^{\infty}} \leq c e^{M_{1} T}\left\|\rho_{0}\right\|_{L^{\infty}} .
$$

It then follows that

$$
\begin{aligned}
& \frac{d}{d t} \int_{\mathbb{S}}\left(y^{2}+y_{x}^{2}+\pi(\rho)^{2}+\pi(\rho)_{x}^{2}+\pi(\rho)_{x x}^{2}\right) d x \\
& \leq c M_{1} \int_{\mathbb{S}}\left(y^{2}+y_{x}^{2}+\pi(\rho)_{x}^{2}+\pi(\rho)_{x x}^{2}\right) d x \\
& \quad+c M_{2} \int_{\mathbb{S}}\left(u_{x x x}^{2}+\pi(\rho)^{2}+\pi(\rho)_{x}^{2}\right) d x \\
& \leq c \int_{\mathbb{S}}\left(y^{2}+y_{x}^{2}+\pi(\rho)^{2}+\pi(\rho)_{x}^{2}+\pi(\rho)_{x x}^{2}\right) d x
\end{aligned}
$$

It is easy to obtain by Gronwall's inequality that

$$
\begin{aligned}
\|u\|_{H^{3}}^{2}+\|\pi(\rho)\|_{H^{2}}^{2} & \leq\|y\|_{H^{1}}^{2}+\|\pi(\rho)\|_{H^{2}}^{2} \\
& \leq e^{c t}\left(\left\|y_{0}\right\|_{H^{1}}^{2}+\left\|\pi\left(\rho_{0}\right)\right\|_{H^{2}}^{2}\right) .
\end{aligned}
$$

Sobolev imbedding, (38), and Theorem 3 ensure that the solution $X$ does not blow up in finite time.

On the other hand, due to the Sobolev imbedding theorem, we observe that $u_{x}(x, t) \rightarrow-\infty$ or $\left\|\rho_{x}(\cdot, t)\right\|_{L^{\infty}} \rightarrow$ $+\infty$ will lead to blow-up of solutions. 
After the local well-posedness of strong solutions (see Theorem 1) is established, a natural question is whether this local solution can exist globally. If the solution only exists in finite time, what induces the blow-up phenomenon? On the other hand, finding sufficient conditions to guarantee the finite time singularities or global existence is very interesting, especially for sufficient conditions added on certain initial data. The following results will give some positive answers.

\section{Blow-Up}

In this section, we pay more attention to the formation of singularities for strong solutions to our system. It will show that wave breaking is one way that singularities arise in smooth solutions. We start this section with the following lemmas.

Lemma 5. Let $X_{0}=\left(u_{0}, \pi\left(\rho_{0}\right)\right) \in H^{s} \times H^{s-1} / \mathbb{R}, s \geq 5 / 2$, and let $T$ be the maximal existence time of the solution $X=$ $(u, \pi(\rho))$ to (7) with the initial data $X_{0}$. Then we obtain the following conservation laws:

$$
\begin{gathered}
E_{1}=\int_{\mathbb{S}}\left(u^{2}+u_{x}^{2}+\pi(\rho)^{2}\right) d x, \\
E_{2}=\int_{\mathbb{S}}\left(u^{3}+\sigma u u_{x}^{2}+u \pi(\rho)^{2}\right) d x .
\end{gathered}
$$

Proof. The proofs are direct consequences of the energy method; similar ones are given in [28] for the two-component Camassa-Holm equations; we refer the readers to [28] for the details.

Remark 6. The conservation of $E_{1}$ guarantees the uniform bound of $u(x, t)$, then Theorem 4 is also interpreted as wave breaking.

Lemma 7 (see [10]). For all $f \in H^{1}(\mathbb{S})$, the following inequality holds:

$$
G *\left(f^{2}+\frac{f_{x}^{2}}{2}\right)(x) \geq C_{0} f^{2}(x),
$$

with

$$
\begin{aligned}
C_{0} & =\frac{1}{2}+\frac{\arctan (\sinh (1 / 2))}{2 \sinh (1 / 2)+2 \arctan (\sinh (1 / 2)) \sinh ^{2}(1 / 2)} \\
& \approx 0.869 .
\end{aligned}
$$

Moreover, $C_{0}$ is the optimal constant obtained by the function $f_{0}$

$$
=\frac{1+\arctan (\sinh (x-[x]-(1 / 2))) \sinh (x-[x]-(1 / 2))}{1+\arctan (\sinh (1 / 2)) \sinh (1 / 2)} .
$$

Lemma 8 (see [33]). For all $f \in H^{1}(\mathbb{S})$, the following inequality holds:

$$
\max _{x \in[0,1]} f^{2}(x) \leq C_{1}\|f\|_{H^{1}(\mathbb{S})}^{2},
$$

where

$$
C_{1}=\frac{e^{1 / 2}+e^{-1 / 2}}{2\left(e^{1 / 2}-e^{-1 / 2}\right)}
$$

Moreover, $C_{1}$ is the minimum value, so in this sense, $C_{1}$ is the optimal constant which is obtained by the associated Green's function

$$
G(x)=\frac{\cosh (x-[x]-(1 / 2))}{2 \sinh (1 / 2)} .
$$

Lemma 9 (see [33]). For any function $f \in H^{2}(\mathbb{S})$, the following inequality holds:

$$
\|f(x)\|_{L^{\infty}(\mathbb{S})}^{2} \leq\left(\int_{\mathbb{S}} f(x) d x\right)^{2}+\|f(x)\|_{H^{1}(\mathbb{S})}^{2} .
$$

Lemma 10 (see [34]). Assume $f(x) \in H^{s}(\mathbb{S}), s>2$. If $\int_{\mathbb{S}} f(x) d x=0$, then

$$
\begin{gathered}
\|f(x)\|_{L^{\infty}(\mathbb{S})}^{2} \leq \frac{1}{12} \int_{\mathbb{S}} f_{x}^{2}(x) d x \\
\int_{\mathbb{S}} f^{2}(x) d x \leq \frac{1}{12} \int_{\mathbb{S}} f_{x}^{2}(x) d x, \\
\int_{\mathbb{S}} f^{2}(x) f_{x}^{2}(x) d x \leq \frac{1}{12}\left(\int_{\mathbb{S}} f_{x}^{2}(x) d x\right)^{2} .
\end{gathered}
$$

It is now to state our result.

Theorem 11. Let $X_{0}=\left(u_{0}, \pi\left(\rho_{0}\right)\right) \in H^{s} \times H^{s-1} / \mathbb{R}, s \geq$ $5 / 2$, and let $T$ be the maximal existence time of solution $X=(u, \pi(\rho))$ to (7) with the initial data $X_{0}$. If the following inequality holds:

$$
\begin{aligned}
& \text { (1) } \int_{\mathbb{S}} u_{0 x}^{3} d x<-\sqrt{\frac{2 E_{1}(0) \kappa}{\sigma}}, \quad \text { if } 0<\sigma<1, \\
& \text { (2) } \int_{\mathbb{S}} u_{0 x}^{3} d x \\
& <-\sqrt{\frac{3(3-\sigma)\left(1-C_{0}\right)}{\sigma} C_{1} E_{1}^{3}(0)+\frac{3}{\sigma} M^{* 2} E_{1}^{2}(0),} \\
& \text { if } 1 \leq \sigma<3,
\end{aligned}
$$

where $E_{1}(0)$ is the initial value of $E_{1}$ in Lemma 5 , and constant $\kappa$ is determined later. Then the corresponding solution $X$ blows up in finite time.

Proof. Differentiating the first equation in system (7) with respect to $x$, we obtain

$$
\begin{aligned}
u_{x t}+ & \sigma\left(u_{x}^{2}+u u_{x x}\right) \\
& +\partial_{x}^{2}\left(G *\left(\frac{3-\sigma}{2} u^{2}+\frac{\sigma}{2} u_{x}^{2}+\frac{1}{2} \pi(\rho)^{2}\right)\right)=0 .
\end{aligned}
$$


Applying the relation $\left(1-\partial_{x}^{2}\right)^{-1} f=G * f$ to (49) gives

$$
\begin{aligned}
u_{x t}+ & \frac{\sigma}{2} u_{x}^{2}+\sigma u u_{x x}+G *\left(\frac{3-\sigma}{2} u^{2}+\frac{\sigma}{2} u_{x}^{2}+\frac{1}{2} \pi(\rho)^{2}\right) \\
& -\frac{3-\sigma}{2} u^{2}-\frac{1}{2} \pi(\rho)^{2}=0
\end{aligned}
$$

If $0<\sigma<1$, multiplying (50) with $u_{x}^{2}$ and integrating by parts subsequently, we obtain

$$
\begin{aligned}
\frac{1}{3} \frac{d}{d t} \int_{\mathbb{S}} u_{x}^{3} d x \\
=-\frac{\sigma}{6} \int_{\mathbb{S}} u_{x}^{4} d x-\int_{\mathbb{S}} u_{x}^{2}\left(G *\left(\frac{3-\sigma}{2} u^{2}+\frac{\sigma}{2} u_{x}^{2}+\frac{1}{2} \pi(\rho)^{2}\right)\right) d x \\
\quad+\int_{\mathbb{S}} u_{x}^{2}\left(\frac{3-\sigma}{2} u^{2}+\frac{1}{2} \pi(\rho)^{2}\right) d x \\
=-\frac{\sigma}{6} \int_{\mathbb{S}} u_{x}^{4} d x-\sigma \int_{\mathbb{S}} u_{x}^{2}\left(G *\left(u^{2}+\frac{u_{x}^{2}}{2}\right)\right) d x \\
\quad-\frac{3-3 \sigma}{2} \int_{\mathbb{S}} u_{x}^{2}\left(G * u^{2}\right) d x \\
\quad-\frac{1}{2} \int_{\mathbb{S}} u_{x}^{2}\left(G * \pi(\rho)^{2}\right) d x+\int_{\mathbb{S}} u_{x}^{2}\left(\frac{3-\sigma}{2} u^{2}+\frac{1}{2} \pi(\rho)^{2}\right) d x \\
\leq-\frac{\sigma}{6} \int_{\mathbb{S}} u_{x}^{4} d x+\frac{3-\sigma-2 \sigma C_{0}}{2} \int_{\mathbb{S}} u_{x}^{2} u^{2} d x+\frac{1}{2} \int_{\mathbb{S}} u_{x}^{2} \pi(\rho)^{2} d x
\end{aligned}
$$

where we have used the following identity:

$$
\int_{\mathbb{S}} u_{x}^{2} u u_{x x} d x=-\frac{1}{3} \int_{\mathbb{S}} u_{x}^{4} d x
$$

Since $3-3 \sigma>0$, and

$$
\begin{gathered}
\int_{\mathbb{S}} u_{x}^{2}\left(G * \pi(\rho)^{2}\right) d x \geq 0, \quad \int_{\mathbb{S}} u_{x}^{2}\left(G * u^{2}\right) d x \geq 0, \\
G *\left(u^{2}+\frac{u_{x}^{2}}{2}\right)(x) \geq C_{0} u^{2}(x),
\end{gathered}
$$

where $C_{0} \approx 0.869<1$.

In the following, we estimate the three terms on the righthand side of (51) one by one. The Cauchy-Schwarz inequality implies that

$$
\left|\int_{\mathbb{S}} u_{x}^{3} d x\right| \leq\left(\int_{\mathbb{S}} u_{x}^{4} d x\right)^{1 / 2}\left(\int_{\mathbb{S}} u_{x}^{2} d x\right)^{1 / 2}
$$

hence

$$
\left|\int_{\mathbb{S}} u_{x}^{4} d x\right| \geq \frac{1}{\int_{\mathbb{S}} u_{x}^{2} d x}\left(\int_{\mathbb{S}} u_{x}^{3} d x\right)^{2} \geq \frac{1}{E_{1}(0)}\left(\int_{\mathbb{S}} u_{x}^{3} d x\right)^{2} .
$$

Using Lemma 8 and the invariant property of $E_{1}$, we have

$$
\int_{\mathbb{S}} u_{x}^{2} u^{2} d x \leq\|u\|_{L^{\infty}}^{2}\left|\int_{\mathbb{S}} u_{x}^{2} d x\right| \leq C_{1} E_{1}^{2}(0) .
$$

Note the fact that

$$
\int_{\mathbb{S}} \pi(\rho) d x=\int_{\mathbb{S}} \rho(x, t) d x-\int_{\mathbb{S}}\left(\int_{\mathbb{S}} \rho(x, t) d x\right) d x=0 .
$$

This implies that for any $t \in[0, T), \pi(\rho)$ has at least a zero point $\eta_{t}$, that is, $\pi\left(\rho\left(\eta_{t}, t\right)\right)=0$. Therefore, we have

$$
\pi(\rho)=\int_{\eta_{t}}^{x} \pi(\rho(s, t))_{s} d s, \quad x \in\left[\eta_{t}, \eta_{t}+1\right] .
$$

Suppose that the solution does not blow-up in finite time, it follows that there exists a constant $M^{*}>0$ such that $u_{x}(x, t)>-M^{*}$ and $\left\|\rho_{x}(\cdot, t)\right\|_{L^{\infty}(\mathbb{S})}$ is bounded by $M^{*}$; thus

$$
|\pi(\rho)|=\left|\int_{\eta_{t}}^{x} \pi(\rho(s, t))_{s} d s\right|=\left|\int_{\eta_{t}}^{x} \rho_{s}(s, t) d s\right| \leq M^{*} .
$$

Then

$$
\left|\int_{\mathbb{S}} u_{x}^{2} \pi(\rho)^{2} d x\right| \leq M^{* 2}\left|\int_{\mathbb{S}} u_{x}^{2} d x\right| \leq M^{* 2} E_{1}(0) .
$$

By (55)-(60), we have

$$
\begin{aligned}
\frac{d}{d t} \int_{\mathbb{S}} u_{x}^{3} d x \leq & -\frac{\sigma}{2} \int_{\mathbb{S}} u_{x}^{4} d x+\frac{3\left(3-\sigma-2 \sigma C_{0}\right)}{2} \int_{\mathbb{S}} u_{x}^{2} u^{2} d x \\
& +\frac{3}{2} \int_{\mathbb{S}} u_{x}^{2} \pi(\rho)^{2} d x \\
\leq & -\frac{\sigma}{2 E_{1}(0)}\left(\int_{\mathbb{S}} u_{x}^{3} d x\right)^{2} \\
& +\frac{3\left(3-\sigma-2 \sigma C_{0}\right)}{2} C_{1} E_{1}^{2}(0)+\frac{3}{2} M^{* 2} E_{1}(0) .
\end{aligned}
$$

For convenience, we denote

$$
\begin{gathered}
\varphi(t)=\int_{\mathbb{S}} u_{x}^{3} d x, \\
\kappa=\frac{3\left(3-\sigma-2 \sigma C_{0}\right)}{2} C_{1} E_{1}^{2}(0)+\frac{3}{2} M^{* 2} E_{1}(0),
\end{gathered}
$$

where we noticed the fact that

$$
3-\sigma-2 \sigma C_{0}>0 \text {. }
$$

That is,

$$
\frac{d \varphi(t)}{d t} \leq-\frac{\sigma}{2 E_{1}(0)} \varphi^{2}(t)+\kappa
$$

Note that if the initial quantity satisfies

$$
\varphi(0)<-\sqrt{\frac{2 E_{1}(0) \kappa}{\sigma}},
$$


the standard argument on the Riccati type inequality and the initial hypothesis ensure that there exists a finite time $T$ such that

$$
\lim _{t \rightarrow T} \int_{\mathbb{S}} u_{x}^{3}(x, t) d x=-\infty
$$

Since

$$
\int_{\mathbb{S}} u_{x}^{3} d x \geq \inf u_{x}(x, t) \int_{\mathbb{S}} u_{x}^{2} d x>\inf u_{x}(x, t) E_{1}(0) .
$$

This implies that

$$
\lim _{t \rightarrow T} \inf u_{x}(x, t)=-\infty .
$$

Then it contradicts the assumption $u_{x}(x, t)>-M^{*}$. By Theorem 4 , we know that the solution must blow up in finite time.

$$
\begin{aligned}
& \text { If } 1 \leq \sigma<3 \text {, we get } \\
& \frac{d}{d t} \int_{\mathbb{S}} u_{x}^{3} d x \\
& =-\frac{\sigma}{2} \int_{\mathbb{S}} u_{x}^{4} d x-\frac{3(3-\sigma)}{2} \int_{\mathbb{S}} u_{x}^{2}\left(G *\left(u^{2}+\frac{u_{x}^{2}}{2}\right)\right) d x \\
& -\frac{9(\sigma-1)}{4} \int_{\mathbb{S}} u_{x}^{2}\left(G * u_{x}^{2}\right) d x \\
& -\frac{1}{2} \int_{\mathbb{S}} u_{x}^{2}\left(G * \pi(\rho)^{2}\right) d x \\
& +3 \int_{\mathbb{S}} u_{x}^{2}\left(\frac{3-\sigma}{2} u^{2}+\frac{1}{2} \pi(\rho)^{2}\right) d x \\
& \leq-\frac{\sigma}{2} \int_{\mathbb{S}} u_{x}^{4} d x+\frac{3(3-\sigma)\left(1-C_{0}\right)}{2} \int_{\mathbb{S}} u_{x}^{2} u^{2} d x \\
& +\frac{3}{2} \int_{\mathbb{S}} u_{x}^{2} \pi(\rho)^{2} d x
\end{aligned}
$$

We also denote that

$$
\varphi(t)=\int_{\mathbb{S}} u_{x}^{3} d x
$$

It follows that

$$
\begin{aligned}
\frac{d \varphi(t)}{d t} \leq & -\frac{\sigma}{2 E_{1}(0)} \varphi^{2}(t)+\frac{3(3-\sigma)\left(1-C_{0}\right)}{2} C_{1} E_{1}^{2}(0) \\
& +\frac{3}{2} M^{* 2} E_{1}(0),
\end{aligned}
$$

where we note $3(3-\sigma)\left(1-C_{0}\right) / 2>0$. Similar to case $(1)$, it is easy to see that under the corresponding condition (2) of our theorem and the previous arguments, blow-up phenomenon occurs. We complete the proof.

As we know, the key issue for partial differential equations lies in the estimates. In the following results, we apply different strategies to derive suitable bounds for solution, then blow-up phenomenon occurs while some special initial values are involved. Precisely, we show the following.
Theorem 12. Suppose that $X_{0}=\left(u_{0}, \pi\left(\rho_{0}\right)\right) \in H^{s} \times H^{s-1} / \mathbb{R}$, $s \geq 5 / 2, u_{0}$ does not vanish identically. If $\int_{\mathbb{S}} u_{0}(x) d x=0$, and one of the following conditions is satisfied

(1) $\sigma \in\left(\frac{3 \sinh (1 / 2)}{6+\sinh (1 / 2)}, 3\right), \quad E_{1}(0)>C_{2}$,

(2) $\sigma \in\left(0, \frac{3 \sinh (1 / 2)}{6+\sinh (1 / 2)}\right]$,

$\varphi(0)$

$$
<-\sqrt{\left(\frac{3-\sigma}{4 \sigma}-\frac{3}{2 \sinh (1 / 2)}\right) E_{1}^{3}(0)+\frac{3}{\sigma} M^{* 2} E_{1}^{2}(0)},
$$

for some constant $C_{2}$ and function $\varphi(t)$ is defined as previously, then the corresponding solution to initial data $X_{0}$ of (7) blows up in finite time.

Proof. Differentiating both sides of the first equation of (7) with respect to variable $x$, we obtain

$$
\begin{aligned}
u_{x t} & +\sigma\left(u_{x}^{2}+u u_{x x}\right) \\
& +\partial_{x}^{2}\left(G *\left(\frac{3-\sigma}{2} u^{2}+\frac{\sigma}{2} u_{x}^{2}+\frac{1}{2} \pi(\rho)^{2}\right)\right)=0 .
\end{aligned}
$$

Applying the relation $\partial_{x}^{2}(G * f)=G * f-f$ to (73) gives

$$
\begin{aligned}
u_{x t} & +\frac{\sigma}{2} u_{x}^{2}+\sigma u u_{x x}+G *\left(\frac{3-\sigma}{2} u^{2}+\frac{\sigma}{2} u_{x}^{2}+\frac{1}{2} \pi(\rho)^{2}\right) \\
& -\frac{3-\sigma}{2} u^{2}-\frac{1}{2} \pi(\rho)^{2}=0 .
\end{aligned}
$$

Multiplying (74) with $u_{x}^{2}$ and integrating by parts subsequently, we obtain

$$
\begin{aligned}
\frac{d}{d t} \int_{\mathbb{S}} u_{x}^{3} d x= & -\frac{\sigma}{2} \int_{\mathbb{S}} u_{x}^{4} d x+\frac{3(3-\sigma)}{2} \int_{\mathbb{S}} u_{x}^{2} u^{2} d x \\
& +\frac{3}{2} \int_{\mathbb{S}} u_{x}^{2} \pi(\rho)^{2} d x \\
& -3 \int_{\mathbb{S}} u_{x}^{2}\left(G * \left(\frac{3-\sigma}{2} u^{2}\right.\right. \\
& \left.\left.+\frac{\sigma}{2} u_{x}^{2}+\frac{1}{2} \pi(\rho)^{2}\right)\right) d x
\end{aligned}
$$

where we have used the following identity:

$$
\int_{\mathbb{S}} u_{x}^{2} u u_{x x} d x=-\frac{1}{3} \int_{\mathbb{S}} u_{x}^{4} d x
$$

On the other hand, we know that $\int_{\mathbb{S}} u(x, t) d x=0$ in view of the hypothesis, and the following inequality holds

$$
\frac{1}{2 \sinh (1 / 2)} \leq G(x) \leq \frac{\cosh (1 / 2)}{2 \sinh (1 / 2)},
$$


where

$$
\frac{\cosh (1 / 2)}{2 \sinh (1 / 2)} \approx 1.082>1
$$

Using Lemma 10 and (77), we obtain

$$
\begin{aligned}
\frac{d}{d t} \int_{\mathbb{S}} u_{x}^{3} d x & \\
\leq & -\frac{\sigma}{2} \int_{\mathbb{S}} u_{x}^{4} d x+\frac{3-\sigma}{8}\left(\int_{\mathbb{S}} u_{x}^{2} d x\right)^{2}+\frac{3}{2} \int_{\mathbb{S}} u_{x}^{2} \pi(\rho)^{2} d x \\
& -\frac{3 \sigma}{2} \int_{\mathbb{S}} u_{x}^{2}\left(G * u_{x}^{2}\right) d x \\
\leq & -\frac{\sigma}{2} \int_{\mathbb{S}} u_{x}^{4} d x+\frac{3-\sigma}{8}\left(\int_{\mathbb{S}} u_{x}^{2} d x\right)^{2} \\
& +\frac{3}{2} \int_{\mathbb{S}} u_{x}^{2} \pi(\rho)^{2} d x-\frac{3 \sigma}{4 \sinh (1 / 2)}\left(\int_{\mathbb{S}} u_{x}^{2} d x\right)^{2} \\
= & -\frac{\sigma}{2} \int_{\mathbb{S}} u_{x}^{4} d x-\left(\frac{3 \sigma}{4 \sinh (1 / 2)}-\frac{3-\sigma}{8}\right)\left(\int_{\mathbb{S}} u_{x}^{2} d x\right)^{2} \\
& +\frac{3}{2} \int_{\mathbb{S}} u_{x}^{2} \pi(\rho)^{2} d x .
\end{aligned}
$$

In view of (60), we obtain that

$$
\begin{aligned}
\frac{d}{d t} \int_{\mathbb{S}} u_{x}^{3} d x \leq & -\frac{\sigma}{2} \int_{\mathbb{S}} u_{x}^{4} d x-\left(\frac{3 \sigma}{4 \sinh (1 / 2)}-\frac{3-\sigma}{8}\right) \\
& \times\left(\int_{\mathbb{S}} u_{x}^{2} d x\right)^{2}+\frac{3}{2} M^{* 2} E_{1}(0) .
\end{aligned}
$$

If $3 \sinh (1 / 2) /(6+\sinh (1 / 2))<\sigma<3$, it is easy to know that

$$
\frac{3 \sigma}{4 \sinh (1 / 2)}-\frac{3-\sigma}{8}>0
$$

If there exists a constant $C_{2}$ such that the initial energy $E_{1}(0)>C_{2}$, then there is some $\delta>0$ such that

$$
\begin{aligned}
& \frac{3}{2} M^{* 2} E_{1}(0) \leq \delta\left(\int_{\mathbb{S}} u_{x}^{2} d x\right)^{2}, \\
& \frac{3 \sigma}{4 \sinh (1 / 2)}-\frac{3-\sigma}{8}-\delta>0 .
\end{aligned}
$$

On the other hand, we have by Lemma 10

$$
\int_{\mathbb{S}} u_{x}^{2} d x \geq \frac{12}{13}\|u\|_{H^{1}(\mathbb{S})}^{2} .
$$

Therefore, the previous inequality yields

$$
\begin{aligned}
\frac{d}{d t} \int_{\mathbb{S}} u_{x}^{3} d x \leq & -\frac{\sigma}{2} \int_{\mathbb{S}} u_{x}^{4} d x \\
& -\frac{144}{169}\left(\frac{3 \sigma}{4 \sinh (1 / 2)}-\frac{3-\sigma}{8}-\delta\right)\|u\|_{H^{1}(\mathbb{S})}^{2} .
\end{aligned}
$$

In view of Hölder's inequality, there holds

$$
\int_{\mathbb{S}} u_{x}^{4} d x \geq\left(\int_{\mathbb{S}} u_{x}^{3} d x\right)^{4 / 3} .
$$

For simplicity of notations, we denote by $\varphi(t)$ and $\mu$ the following quantities:

$$
\int_{\mathbb{S}} u_{x}^{3} d x, \quad \frac{144}{169}\left(\frac{3 \sigma}{4 \sinh (1 / 2)}-\frac{3-\sigma}{8}-\delta\right)
$$

respectively. Therefore we have

$$
\frac{d \varphi(t)}{d t} \leq-\frac{\sigma}{2} \varphi^{4 / 3}(t)-\mu\|u\|_{H^{1}(\mathbb{S})}^{4}
$$

First, we can easily get $\varphi(t) \leq \varphi(0)-\mu\|u\|_{H^{1}(\mathbb{S})}^{4} t$, and it is not difficult to find that there exists a $t_{0} \geq 0$ such that $\varphi\left(t_{0}\right)<0$. Then for $t>t_{0}$, we get

$$
\frac{d \varphi(t)}{d t} \leq-\frac{\sigma}{2} \varphi^{4 / 3}(t), \quad \text { with } \varphi\left(t_{0}\right)<0 .
$$

Solving this inequality yields

$$
\varphi(t) \leq\left(\varphi^{-1 / 3}\left(t_{0}\right)+\frac{\sigma}{6}\left(t-t_{0}\right)\right)^{-3},
$$

which approaches $-\infty$ as $t$ arrives at $T_{0}=-(6 / \sigma) \varphi^{-1 / 3}\left(t_{0}\right)+t_{0}$; that is, there exists a time $T \leq-(6 / \sigma) \varphi^{-1 / 3}\left(t_{0}\right)+t_{0}$ such that

$$
\lim _{t \rightarrow T} \int_{\mathbb{S}} u_{x}^{3} d x=-\infty
$$

Therefore, it follows that

$$
\liminf _{t \rightarrow T x \in \mathbb{R}} u_{x}(x, t)=-\infty .
$$

Then it contradicts the assumption $u_{x}(x, t)>-M^{*}$. By blowup scenario, we know that the solution must blow up in finite time.

If $0<\sigma \leq 3 \sinh (1 / 2) /(6+\sinh (1 / 2))$, then we have

$$
\begin{aligned}
\frac{d}{d t} \int_{\mathbb{S}} u_{x}^{3} d x \leq & -\frac{\sigma}{2} \int_{\mathbb{S}} u_{x}^{4} d x \\
& +\left(\frac{3-\sigma}{8}-\frac{3 \sigma}{4 \sinh (1 / 2)}\right) \\
& \times\left(\int_{\mathbb{S}} u_{x}^{2} d x\right)^{2}+\frac{3}{2} M^{* 2} E_{1}(0),
\end{aligned}
$$

where

$$
\frac{3-\sigma}{8}-\frac{3 \sigma}{4 \sinh (1 / 2)} \geq 0
$$

We also use $\varphi(t)$ as previously to get

$$
\begin{aligned}
\frac{d \varphi(t)}{d t} \leq & -\frac{\sigma}{2 E_{1}(0)} \varphi^{2}(t) \\
& +\left(\frac{3-\sigma}{8}-\frac{3 \sigma}{4 \sinh (1 / 2)}\right) E_{1}^{2}(0)+\frac{3}{2} M^{* 2} E_{1}(0) .
\end{aligned}
$$

Similar arguments to (64) in Theorem 11 and condition (2) guarantee the finite time blow-up of solution to (7). 
The zero mean of $u(x, t)$ in the previous theorem can be substituted by $E_{2}(0)=0$; blow-up still occurs with the aid of different estimate from (83).

Theorem 13. Assume that $X_{0}=\left(u_{0}, \pi\left(\rho_{0}\right)\right) \in H^{s} \times H^{s-1} / \mathbb{R}$, $s \geq 5 / 2, u_{0}$ does not vanish identically, $\int_{\mathbb{S}}\left(u_{0}^{3}+\sigma u_{0} u_{0 x}^{2}+\right.$ $\left.u_{0} \pi\left(\rho_{0}\right)^{2}\right) d x=0$, if one of the following conditions is satisfied:

(1) $\sigma \in\left(\frac{3 \sinh (1 / 2)}{2+\sinh (1 / 2)}, 3\right), \quad E_{1}(0)>C_{3}$,

(2) $\sigma \in\left(0, \frac{3 \sinh (1 / 2)}{2+\sinh (1 / 2)}\right]$,

$\varphi(0)$

$$
<-\sqrt{\left(\frac{3(3-\sigma)}{4 \sigma}-\frac{3}{2 \sinh (1 / 2)}\right) E_{1}^{3}(0)+\frac{3}{\sigma} M^{* 2} E_{1}^{2}(0)},
$$

for some constant $C_{3}$, then the corresponding solution to (7) blows up in finite time.

Proof. By assumption and the invariance property of $E_{2}$, we have

$$
\begin{aligned}
\int_{\mathbb{S}} u & \left(u^{2}+\sigma u_{x}^{2}+\pi(\rho)^{2}\right) d x \\
& =\int_{\mathbb{S}}\left(u^{3}+\sigma u u_{x}^{2}+u \pi(\rho)^{2}\right) d x=0 .
\end{aligned}
$$

Therefore, $u(x, t)$ must change sign, so there exists at least one zero point on $\mathbb{S}$. Then for each $t \in[0, T)$, suppose that there is a $\xi_{t} \in[0,1]$ such that $u\left(\xi_{t}, t\right)=0$, for $x \in \mathbb{S}$ we have

$$
\begin{aligned}
u^{2}(x, t) & =\left(\int_{\xi_{t}}^{x} u_{x} d x\right)^{2} \\
& \leq\left(x-\xi_{t}\right) \int_{\xi_{t}}^{x} u_{x}^{2} d x, \quad x \in\left[\xi_{t}, \xi_{t}+\frac{1}{2}\right] .
\end{aligned}
$$

Thus, the previous relation and an integration by parts yield

$$
\begin{aligned}
\int_{\xi_{t}}^{\xi_{t}+1 / 2} u^{2} u_{x}^{2} d x \leq & \int_{\xi_{t}}^{\xi_{t}+1 / 2}\left(x-\xi_{t}\right) u_{x}^{2}\left(\int_{\xi_{t}}^{x} u_{x}^{2} d z\right) d x \\
= & \int_{\xi_{t}}^{\xi_{t}+1 / 2}\left(x-\xi_{t}\right)\left(\int_{\xi_{t}}^{x} u_{x}^{2} d z\right) d\left(\int_{\xi_{t}}^{x} u_{x}^{2} d z\right) \\
= & \frac{1}{4}\left(\int_{\xi_{t}}^{\xi_{t}+1 / 2} u_{x}^{2} d z\right)^{2} \\
& -\frac{1}{2} \int_{\xi_{t}}^{\xi_{t}+1 / 2}\left(\int_{\xi_{t}}^{x} u_{x}^{2} d z\right)^{2} d x \\
\leq & \frac{1}{4}\left(\int_{\xi_{t}}^{\xi_{t}+1 / 2} u_{x}^{2} d z\right)^{2} .
\end{aligned}
$$

Doing a similar estimate on $\left[\xi_{t}+1 / 2, \xi_{t}+1\right]$, we obtain

$$
\int_{\mathbb{S}} u^{2} u_{x}^{2} d x \leq \frac{1}{4}\left(\int_{\mathbb{S}} u_{x}^{2} d x\right)^{2}
$$

In view of (97), we also have

$$
\|u(x, t)\|_{L^{\infty}(\mathbb{S})}^{2} \leq \frac{1}{2} \int_{\mathbb{S}} u_{x}^{2} d x
$$

Let

$$
\varphi(t)=\int_{\mathbb{S}} u_{x}^{3} d x, \quad t \geq 0,
$$

we obtain

$$
\begin{aligned}
\frac{d \varphi(t)}{d t}= & -\frac{\sigma}{2} \int_{\mathbb{S}} u_{x}^{4} d x \\
& +\frac{3(3-\sigma)}{2} \int_{\mathbb{S}} u_{x}^{2} u^{2} d x+\frac{3}{2} \int_{\mathbb{S}} u_{x}^{2} \pi(\rho)^{2} d x \\
& -3 \int_{\mathbb{S}} u_{x}^{2}\left(G *\left(\frac{3-\sigma}{2} u^{2}+\frac{\sigma}{2} u_{x}^{2}+\frac{1}{2} \pi(\rho)^{2}\right)\right) d x \\
\leq & -\frac{\sigma}{2} \int_{\mathbb{S}} u_{x}^{4} d x-\frac{1}{4}\left(\frac{3 \sigma}{\sinh (1 / 2)}-\frac{3(3-\sigma)}{2}\right) \\
& \times\left(\int_{\mathbb{S}} u_{x}^{2} d x\right)^{2} \\
& +\frac{3}{2} \int_{\mathbb{S}} u_{x}^{2} \pi(\rho)^{2} d x,
\end{aligned}
$$

where we use the fact $1 / 2 \sinh (1 / 2) \leq G(x) \leq \cosh (1 / 2) /$ $2 \sinh (1 / 2)$, then

$$
\begin{aligned}
\int_{\mathbb{S}} u_{x}^{2}\left(G * u_{x}^{2}\right) d x & \leq\left\|G * u_{x}^{2}\right\|_{L^{\infty}(\mathbb{S})} \\
& \leq \frac{1}{2 \sinh (1 / 2)}\left(\int_{\mathbb{S}} u_{x}^{2} d x\right)^{2}
\end{aligned}
$$

For $\sigma \in(3 \sinh (1 / 2) /(2+\sinh (1 / 2)), 3)$, we have

$$
\frac{3 \sigma}{\sinh (1 / 2)}-\frac{3(3-\sigma)}{2} \geq 0 .
$$

If there is a constant $C_{3}$ such that the initial energy $E_{1}(0)>$ $C_{3}$, there exists some $\delta>0$ such that

$$
\begin{aligned}
& \frac{3}{2} M^{* 2} E_{1}(0) \leq \frac{\delta}{4}\left(\int_{\mathbb{S}} u_{x}^{2} d x\right)^{2}, \\
& \frac{3 \sigma}{\sinh (1 / 2)}-\frac{3(3-\sigma)}{2}-\delta>0 .
\end{aligned}
$$


On the other hand, we have $\int_{\mathbb{S}} u_{x}^{2} d x \geq(2 / 3)\|u\|_{H^{1}(\mathbb{S})}^{2}$. Therefore, the previous inequality yields

$$
\begin{aligned}
\frac{d \varphi(t)}{d t} \leq & -\frac{\sigma}{2} \int_{\mathbb{S}} u_{x}^{4} d x-\frac{1}{9}\left(\frac{3 \sigma}{\sinh (1 / 2)}-\frac{3(3-\sigma)}{2}-\delta\right) \\
& \times\|u\|_{H^{1}(\mathbb{S})}^{4} \\
\leq & -\frac{\sigma}{2} \varphi^{4 / 3}(t)-\frac{1}{9}\left(\frac{3 \sigma}{\sinh (1 / 2)}-\frac{3(3-\sigma)}{2}-\delta\right) \\
& \times\|u\|_{H^{1}(\mathbb{S})}^{4} .
\end{aligned}
$$

The remaining part is very close to Theorem 12 , so we omit it. So the solution must blow up in finite time.

For $\sigma \in(0,3 \sinh (1 / 2) /(2+\sinh (1 / 2))]$, then

$$
\begin{aligned}
\frac{d \varphi(t)}{d t} \leq & -\frac{\sigma}{2 E_{1}(0)} \varphi^{2}(t) \\
& +\left(\frac{3(3-\sigma)}{8}-\frac{3 \sigma}{4 \sinh (1 / 2)}\right) E_{1}^{2}(0) \\
& +\frac{3}{2} M^{* 2} E_{1}(0)
\end{aligned}
$$

where $3(3-\sigma) / 8-3 \sigma / 4 \sinh (1 / 2) \geq 0$. Note that if the initial quantity satisfies

$$
\varphi(0)<-\sqrt{\left(\frac{3(3-\sigma)}{4 \sigma}-\frac{3}{2 \sinh (1 / 2)}\right) E_{1}^{3}(0)+\frac{3}{\sigma} M^{* 2} E_{1}^{2}(0)},
$$

then condition (2) can conclude that the solution to (7) goes to $-\infty$ in finite time. This completes the proof.

Theorem 14. Suppose that $X_{0}=\left(u_{0}, \pi\left(\rho_{0}\right)\right) \in H^{s} \times$ $H^{s-1} / \mathbb{R}, s \geq 5 / 2, X=(u, \pi(\rho))$ is the solution to system (7) with the initial data $X_{0}$. If there is some point $x_{0} \in \mathbb{S}$ such that one of the following conditions is satisfied:

(1) $u_{0}^{\prime}\left(x_{0}\right)<-\sqrt{\frac{3-\sigma-2 \sigma C_{0}}{\sigma} C_{1} E_{1}(0)}, \quad 0<\sigma<1$,

(2) $u_{0}^{\prime}\left(x_{0}\right)<-\sqrt{\frac{3-\sigma}{\sigma}\left(1-C_{0}\right) C_{1} E_{1}(0)}, \quad 1 \leq \sigma<3$,

where the constants $C_{0}$ and $C_{1}$ are given in Lemmas 7 and 8 , $E_{1}(0)$ is the initial value of $E_{1}$, then the solution $X$ must blow up in finite time.

Proof. Differentiating the first equation in system (7) with respect to $x$ and noticing that $\partial_{x}^{2}(G * f)=G * f-f$, we have

$$
\begin{aligned}
u_{x t} & +\frac{\sigma}{2} u_{x}^{2}+\sigma u u_{x x}+G *\left(\frac{3-\sigma}{2} u^{2}+\frac{\sigma}{2} u_{x}^{2}+\frac{1}{2} \pi(\rho)^{2}\right) \\
& -\frac{3-\sigma}{2} u^{2}-\frac{1}{2} \pi(\rho)^{2}=0 .
\end{aligned}
$$

When $0<\sigma<1$, this equation, in combination with (11), yields

$$
\begin{aligned}
& \frac{d}{d t} u_{x}\left(q_{2}(x, t), t\right) \\
& =\left(u_{x t}+\sigma u u_{x x}\right)\left(q_{2}, t\right) \\
& =\left(-\frac{\sigma}{2} u_{x}^{2}+\frac{3-\sigma}{2} u^{2}+\frac{1}{2} \pi(\rho)^{2}\right. \\
& \left.\quad-G *\left(\frac{3-\sigma}{2} u^{2}+\frac{\sigma}{2} u_{x}^{2}+\frac{1}{2} \pi(\rho)^{2}\right)\right)\left(q_{2}, t\right) \\
& \leq\left(-\frac{\sigma}{2} u_{x}^{2}+\frac{3-\sigma}{2} u^{2}+\frac{1}{2} \pi(\rho)^{2}\right. \\
& \left.\quad-\sigma G *\left(u^{2}+\frac{u_{x}^{2}}{2}\right)-\frac{3-3 \sigma}{2} G * u^{2}\right)\left(q_{2}, t\right)
\end{aligned}
$$

Note that

$$
\int_{\mathbb{S}} \pi(\rho) d x=0
$$

We can deduce that there exists at least one point $x_{0}$ such that $\pi\left(\rho\left(q_{2}\left(x_{0}, t\right), t\right)\right)=0$ for $t \in[0, T)$. Let us consider this problem at $\left(q_{2}\left(x_{0}, t\right), t\right)$. For convenience, we denote $u_{x}\left(q_{2}\left(x_{0}, t\right), t\right)=m(t)$. Then we have

$$
\begin{aligned}
\frac{d m(t)}{d t} & \leq-\frac{\sigma}{2} m^{2}(t)+\frac{3-\sigma}{2} u^{2}-\sigma G *\left(u^{2}+\frac{u_{x}^{2}}{2}\right) \\
& \leq-\frac{\sigma}{2} m^{2}(t)+\frac{3-\sigma-2 \sigma C_{0}}{2} C_{1} E_{1}(0) .
\end{aligned}
$$

Using the notation $\varrho=\left(3-\sigma-2 \sigma C_{0} / \sigma\right) C_{1} E_{1}(0)$, where $3-$ $\sigma-2 \sigma C_{0}>0$, we have

$$
\frac{d m(t)}{d t} \leq-\frac{\sigma}{2}\left(m^{2}(t)-\varrho\right) .
$$

In view of the initial condition, it is not difficult to obtain

$$
\frac{d m(t)}{d t} \leq \frac{\sigma(\delta-1)}{2} m^{2}(t)
$$

with $0<\delta<1$ determined by $\delta m^{2}(0)=\varrho$. Then, by using the standard arguments for this type of inequality and our hypothesis, it is easy to conclude that the lifespan of the solution is finite; that is, blow-up phenomenon occurs.

When $1 \leq \sigma<3$, then

$$
\begin{aligned}
\frac{d m(t)}{d t} \leq & -\frac{\sigma}{2} u_{x}^{2}+\frac{3-\sigma}{2} u^{2}-\frac{3-\sigma}{2} G *\left(u^{2}+\frac{u_{x}^{2}}{2}\right) \\
& -\frac{3 \sigma-3}{4}\left(G * u_{x}^{2}\right) \\
\leq & -\frac{\sigma}{2} u_{x}^{2}+\frac{3-\sigma}{2}\left(1-C_{0}\right) u^{2} \\
\leq & -\frac{\sigma}{2} m^{2}(t)+\frac{3-\sigma}{2}\left(1-C_{0}\right) C_{1} E_{1}(0),
\end{aligned}
$$


where $(3-\sigma)\left(1-C_{0}\right)>0$. Similar to the arguments about (113), under the condition (2), the corresponding solution $X$ blows up in finite time. We complete the proof.

Theorem 15. Suppose that $X_{0}=\left(u_{0}, \pi\left(\rho_{0}\right)\right) \in H^{s} \times$ $H^{s-1} / \mathbb{R}, s \geq 5 / 2, X=(u, \pi(\rho))$ is the solution to system (7) with the initial data $X_{0}$. If there holds one of the following conditions for some point $x_{0} \in \mathbb{S}$

$$
\begin{array}{ll}
\text { (1) } u_{0}^{\prime}\left(x_{0}\right)<-\sqrt{\frac{3-\sigma-2 \sigma C_{0}}{\sigma} K(0)}, & 0<\sigma<1, \\
\text { (2) } u_{0}^{\prime}\left(x_{0}\right)<-\sqrt{\frac{3-\sigma}{\sigma}\left(1-C_{0}\right) K(0)}, & 1 \leq \sigma<3
\end{array}
$$

where the constant $C_{0}$ is the best constant given by Lemma 7 and

$$
K(0)=\left(\int_{\mathbb{S}} u_{0}(x) d x\right)^{2}+\int_{\mathbb{S}}\left(u_{0}^{2}+u_{0 x}^{2}+\pi\left(\rho_{0}\right)^{2}\right) d x
$$

Let $T$ be the maximal existence time of the corresponding solution to (7) with the initial data $X_{0}$. Then $T$ is finite.

Proof. We easily know that $\int_{\mathbb{S}} u(x, t) d x$ is also an invariant with respect to time. The result follows by using Lemma 9 that

$$
\begin{aligned}
\|u(x)\|_{L^{\infty}(\mathbb{S})}^{2} & \leq\left(\int_{\mathbb{S}} u(x) d x\right)^{2}+\|u(x)\|_{H^{1}(\mathbb{S})}^{2} \\
& <\left(\int_{\mathbb{S}} u_{0}(x) d x\right)^{2}+E_{0}=K_{0},
\end{aligned}
$$

in (113) instead of $\|u(x)\|_{L^{\infty}(\mathbb{S})}^{2} \leq C_{1}\|u(x)\|_{H^{1}(\mathbb{S})}^{2} \leq C_{1} E_{1}(0)$.

In the following, as a byproduct, we examine the blow-up rate while the solution blows up in finite time.

Theorem 16. Assume that $X_{0}=\left(u_{0}, \pi\left(\rho_{0}\right)\right) \in H^{s} \times$ $H^{s-1} / \mathbb{R}, s \geq 5 / 2, X=(u, \pi(\rho))$ is the corresponding solution with the initial data $X_{0}$. If there holds the condition of Theorem 14, then we have the following description:

$$
\lim _{t \rightarrow T}(T-t) m(t)=-\frac{2}{\sigma}
$$

where $\sigma>0$ and $m(t)$ is defined in Theorem 14 .

Proof. The conclusion follows from the theory of ordinary differential equations to inequality (113). Indeed, when $0<$ $\sigma<1$, by (113) we have

$$
\frac{d m(t)}{d t} \leq-\frac{\sigma}{2} m^{2}(t)+\frac{3-\sigma-2 \sigma C_{0}}{2} C_{1} E_{1}(0) .
$$

In view of Lemma 8 and the conservation of $E_{1}$, there holds for all $t \in[0, T)$ that

$$
\left|\frac{d m(t)}{d t}+\frac{\sigma}{2} m^{2}(t)\right| \leq \frac{\sigma \varrho}{2} .
$$

It follows that

$$
-\frac{\sigma \varrho}{2} \leq \frac{d m(t)}{d t}+\frac{\sigma}{2} m^{2}(t) \leq \frac{\sigma \varrho}{2}, \quad \text { a.e. on }(0, T) .
$$

Since $\lim _{t \rightarrow T} m(t)=-\infty$ by Theorem 14, it implies that for any $\varepsilon \in(0, \sigma / 2)$ there exists a $t_{0}$ such that $m^{2}(t)>\sigma \varrho / 2 \varepsilon$ for all $t \in\left[t_{0}, T\right)$. Therefore,

$$
-m^{2}(t) \varepsilon \leq \frac{d m(t)}{d t}+\frac{\sigma}{2} m^{2}(t) \leq m^{2}(t) \varepsilon,
$$

that is,

$$
-\frac{\sigma}{2}-\varepsilon \leq \frac{1}{m^{2}(t)} \frac{d m(t)}{d t} \leq-\frac{\sigma}{2}+\varepsilon
$$

Direct integration from $t$ to $T$ gives

$$
-\frac{\sigma}{2}-\varepsilon \leq \frac{1}{(T-t) m(t)} \leq-\frac{\sigma}{2}+\varepsilon,
$$

and the arbitrariness of $\varepsilon$ leads to our result.

When $1 \leq \sigma<3$, by (116) we obtain

$$
\frac{d m(t)}{d t} \leq-\frac{\sigma}{2} m^{2}(t)+\frac{\sigma \omega}{2}
$$

where $\omega=((3-\sigma) / \sigma)\left(1-C_{0}\right) C_{1} E_{1}(0)$. Similar to the arguments for $0<\sigma<1$, we can get the same result.

\section{Acknowledgments}

This work was partially supported by Natural Science Foundation of China (Grant no. 11226172), Zhejiang Provincial Natural Science Foundation of China (Grant nos. LQ12A01009 and LY12A01014).

\section{References}

[1] G. M. Coclite, H. Holden, and K. H. Karlsen, "Global weak solutions to a generalized hyperelastic-rod wave equation," SIAM Journal on Mathematical Analysis, vol. 37, no. 4, pp. 10441069, 2005.

[2] H.-H. Dai, "Model equations for nonlinear dispersive waves in a compressible Mooney-Rivlin rod," Acta Mechanica, vol. 127, no. 1-4, pp. 193-207, 1998.

[3] Z. Guo and Y. Zhou, "Wave breaking and persistence properties for the dispersive rod equation," SIAM Journal on Mathematical Analysis, vol. 40, no. 6, pp. 2567-2580, 2009.

[4] H. Holden and X. Raynaud, "Global conservative solutions of the generalized hyperelastic-rod wave equation," Journal of Differential Equations, vol. 233, no. 2, pp. 448-484, 2007.

[5] L. Jin, Y. Liu, and Y. Zhou, "Blow-up of solutions to a periodic nonlinear dispersive rod equation," Documenta Mathematica, vol. 15, pp. 267-283, 2010.

[6] Y.-M. Liu and Y. Zhou, "Blow-up phenomenon for a periodic rod equation," Journal of Physics A, vol. 41, no. 34, Article ID 344013, 10 pages, 2008.

[7] O. G. Mustafa, "Global conservative solutions of the hyperelastic rod equation," International Mathematics Research Notices, vol. 2007, no. 13, 2007. 
[8] E. Wahlén, "On the blow-up of solutions to a nonlinear dispersive rod equation," Journal of Mathematical Analysis and Applications, vol. 323, no. 2, pp. 1318-1324, 2006.

[9] Z. Yin, "On the blow-up of solutions of a periodic nonlinear dispersive wave equation in compressible elastic rods," Journal of Mathematical Analysis and Applications, vol. 288, no. 1, pp. 232-245, 2003.

[10] Y. Zhou, "Blow-up of solutions to a nonlinear dispersive rod equation," Calculus of Variations and Partial Differential Equations, vol. 25, no. 1, pp. 63-77, 2006.

[11] Y. Zhou, "Local well-posedness and blow-up criteria of solutions for a rod equation," Mathematische Nachrichten, vol. 278, no. 14, pp. 1726-1739, 2005.

[12] Y. Zhou, "Blow-up phenomenon for a periodic rod equation," Physics Letters A, vol. 353, no. 6, pp. 479-486, 2006.

[13] A. Bressan and A. Constantin, "Global conservative solutions of the Camassa-Holm equation," Archive for Rational Mechanics and Analysis, vol. 183, no. 2, pp. 215-239, 2007.

[14] A. Bressan and A. Constantin, "Global dissipative solutions of the Camassa-Holm equation," Analysis and Applications, vol. 5, no. 1, pp. 1-27, 2007.

[15] R. Camassa and D. D. Holm, "An integrable shallow water equation with peaked solitons," Physical Review Letters, vol. 71, no. 11, pp. 1661-1664, 1993.

[16] A. Constantin and J. Escher, "Well-posedness, global existence, and blowup phenomena for a periodic quasi-linear hyperbolic equation," Communications on Pure and Applied Mathematics, vol. 51, no. 5, pp. 475-504, 1998.

[17] H. Holden and X. Raynaud, "Global conservative solutions of the Camassa-Holm equation-a Lagrangian point of view," Communications in Partial Differential Equations, vol. 32, no. 10-12, pp. 1511-1549, 2007.

[18] H. Holden and X. Raynaud, "Dissipative solutions for the Camassa-Holm equation," Discrete and Continuous Dynamical Systems A, vol. 24, no. 4, pp. 1047-1112, 2009.

[19] Z. Jiang, L. Ni, and Y. Zhou, "Wave breaking of the CamassaHolm equation," Journal of Nonlinear Science, vol. 22, no. 2, pp. 235-245, 2012.

[20] Z. Xin and P. Zhang, "On the weak solutions to a shallow water equation," Communications on Pure and Applied Mathematics, vol. 53, no. 11, pp. 1411-1433, 2000.

[21] Y. Zhou, "Wave breaking for a shallow water equation," Nonlinear Analysis: Theory, Methods \& Applications, vol. 57, no. 1, pp. 137-152, 2004.

[22] R. M. Chen and Y. Liu, "Wave breaking and global existence for a generalized two-component Camassa-Holm system," International Mathematics Research Notices, vol. 2011, no. 6, pp. 13811416, 2011.

[23] A. Constantin and R. I. Ivanov, "On an integrable twocomponent Camassa-Holm shallow water system," Physics Letters A, vol. 372, no. 48, pp. 7129-7132, 2008.

[24] M. Chen, S.-Q. Liu, and Y. Zhang, "A two-component generalization of the Camassa-Holm equation and its solutions," Letters in Mathematical Physics, vol. 75, no. 1, pp. 1-15, 2006.

[25] J. Escher, O. Lechtenfeld, and Z. Yin, "Well-posedness and blowup phenomena for the 2-component Camassa-Holm equation," Discrete and Continuous Dynamical Systems A, vol. 19, no. 3, pp. 493-513, 2007.

[26] G. Falqui, "On a Camassa-Holm type equation with two dependent variables," Journal of Physics A, vol. 39, no. 2, pp. 327342, 2006.
[27] G. Gui and Y. Liu, "On the global existence and wave-breaking criteria for the two-component Camassa-Holm system," Journal of Functional Analysis, vol. 258, no. 12, pp. 4251-4278, 2010.

[28] Z. Guo and Y. Zhou, "On solutions to a two-component generalized Camassa-Holm equation," Studies in Applied Mathematics, vol. 124, no. 3, pp. 307-322, 2010.

[29] Z. Guo, "Blow-up and global solutions to a new integrable model with two components," Journal of Mathematical Analysis and Applications, vol. 372, no. 1, pp. 316-327, 2010.

[30] M. Yuen, "Self-similar blowup solutions to the 2-component Camassa-Holm equations," Journal of Mathematical Physics, vol. 51, no. 9, Article ID 093524, 14 pages, 2010.

[31] Z. Guo, L. Jin, and L. Ni, "Blow-up criteria of solutions for a modified two-component hyperelastic rod system," Journal of Mathematical Physics, vol. 53, Article ID 123501, 15 pages, 2012.

[32] M. Kohlmann, "On a two-component $\pi$-Camassa-Holm system," Journal of Geometry and Physics, vol. 62, no. 4, pp. 832838, 2012.

[33] Y. Zhou, "Wave breaking for a periodic shallow water equation," Journal of Mathematical Analysis and Applications, vol. 290, no. 2, pp. 591-604, 2004.

[34] Y. Zhou and Z. Guo, "Blow up and propagation speed of solutions to the DGH equation," Discrete and Continuous Dynamical Systems B, vol. 12, no. 3, pp. 657-670, 2009. 


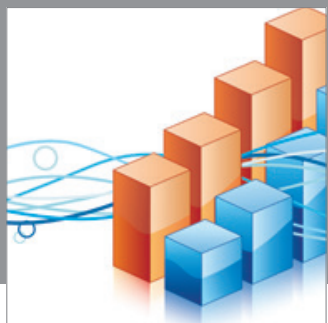

Advances in

Operations Research

mansans

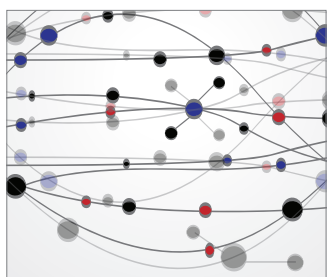

The Scientific World Journal
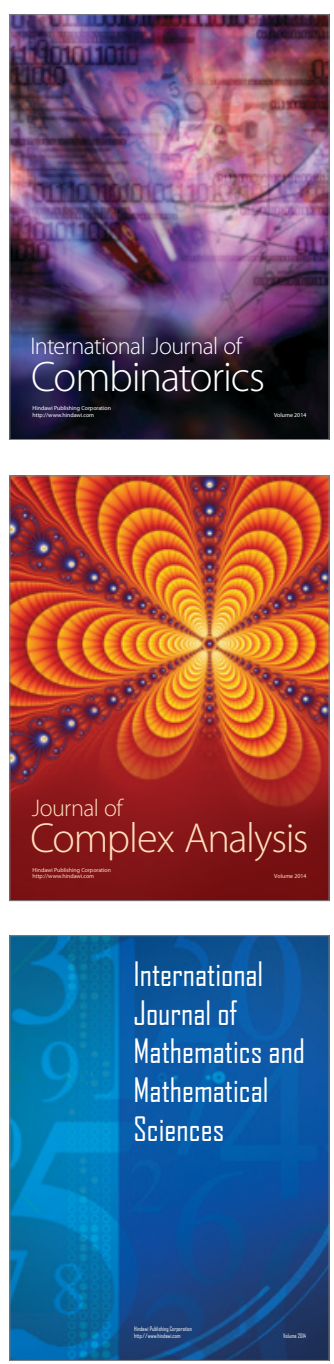
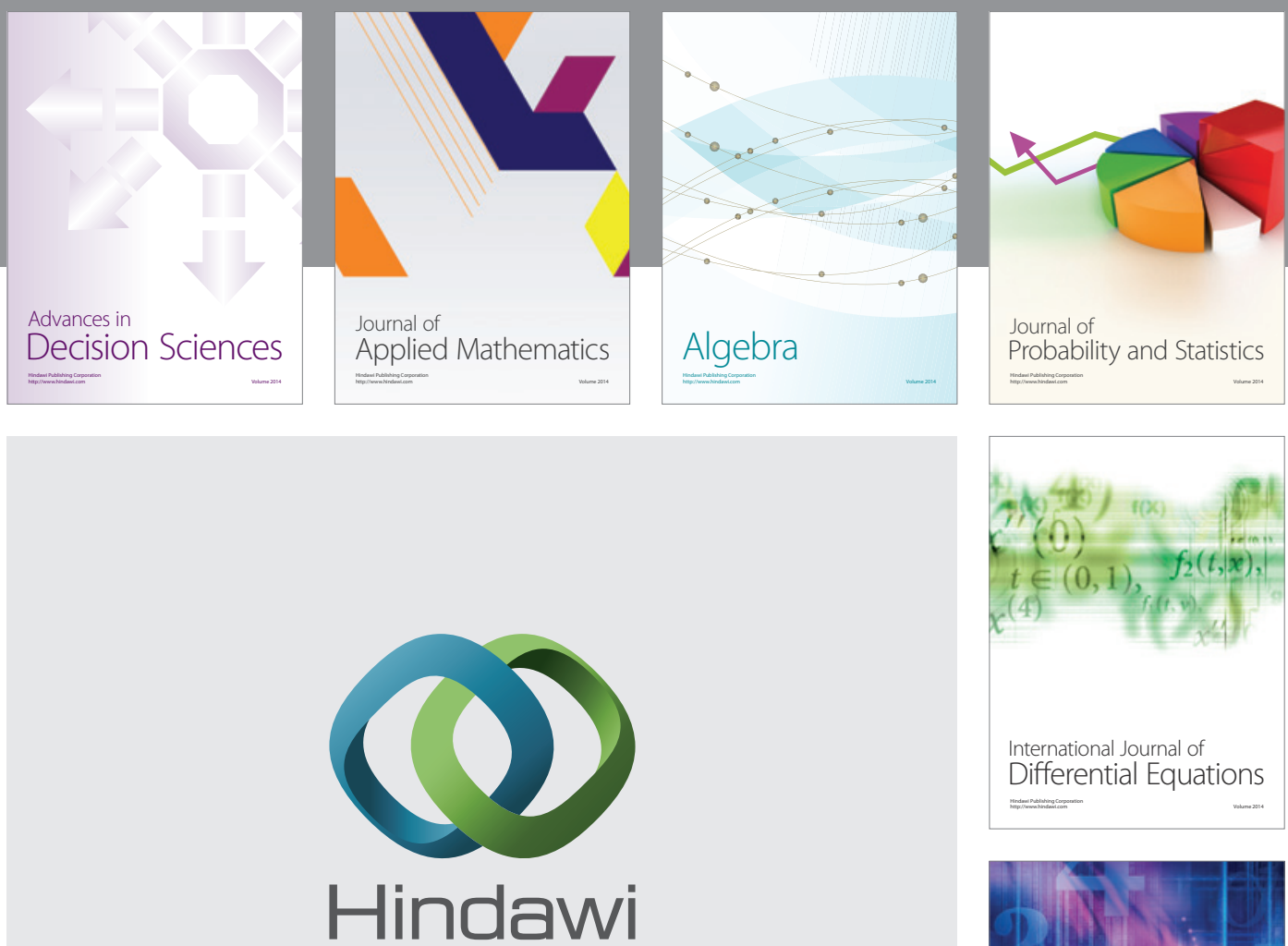

Submit your manuscripts at http://www.hindawi.com
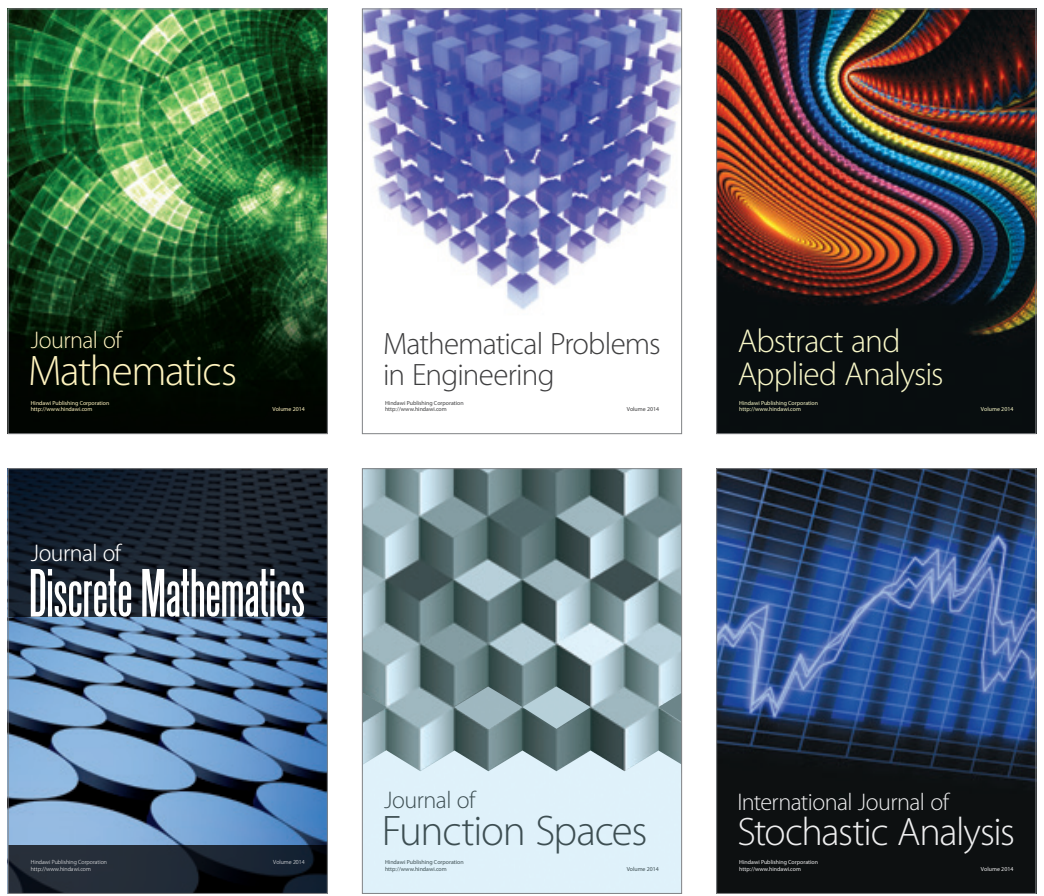

Journal of

Function Spaces

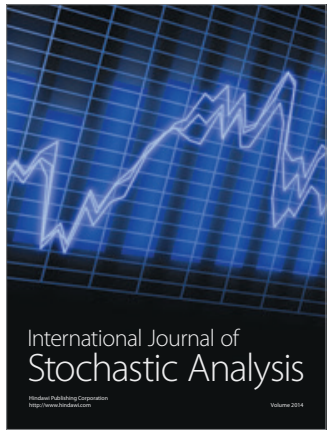

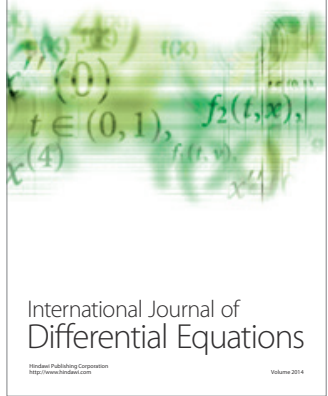
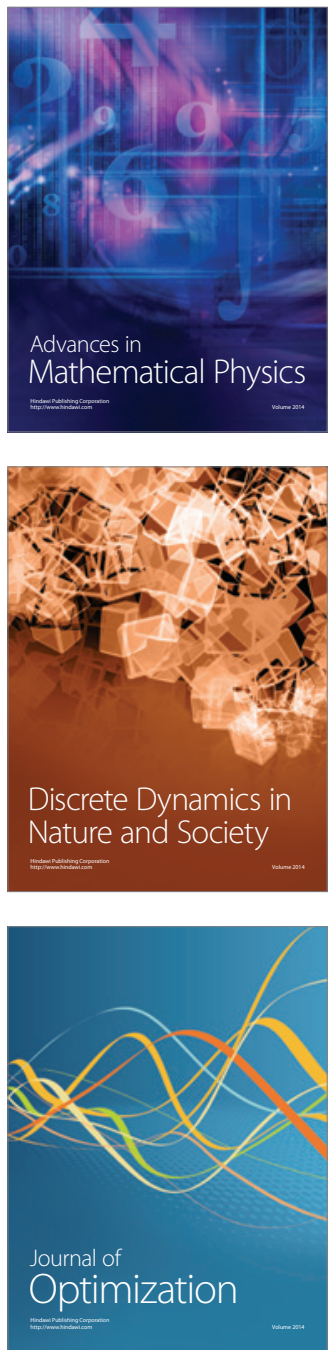